\title{
Asymmetry Theory derived from the principle of constant light speed
}

\author{
Revised 2/18/2021 14:16:00
}

\author{
Qian Chen \\ International Education Foundation \\ 4667 Highland Ct, Grandville, MI, USA 49418 \\ e-mail: georgechen@iefstudy.org
}

\begin{abstract}
"The principle of the constancy of the velocity of light" was well established, while the further assumption that the light velocity is independent of the motion of the observer was never directly proven by any experiment. Based solely on this principle without any unproven assumptions, a comprehensive theoretic framework of the electrodynamics of moving bodies, named "Asymmetry Theory", is derived purely through strict mathematics. A formula of the light velocity was mathematically derived, which is proven by the Sagnac effect and provides mathematical explanations for one-way light speed measurement, stellar aberration, and the M-M experiment. Other mathematically derived results include:

1. A formula for observed "time dilation", which resolves the "twin paradox".

2. Doppler Effect is simply a phenomenon of observed "time dilation" and one general formula covers traditional and transverse Doppler Effects, cosmological redshift, and time-varying velocities.

3. Lorentz force law is invariant under Galilean transformation, with the correct definition of velocity following Barnett's experiment explanation.

4. A generalized form of Maxwell wave equations derived from the original equations, which is covariant under Galilean transformation.

5. The electrodynamics including particle acceleration and Mass-Energy relationship.

Asymmetry Theory is comprehensive, self-consistent and in harmony with all existing experiments. It provides straightforward and mathematical explanations of key phenomenons without any paradox. Furthermore, Maxwell's equations provide it the theoretic base and proof. Based on its predictions, two experiment designs are proposed for further conclusive confirmation.
\end{abstract}

Keywords: Light velocity, Special Relativity, Asymmetry Theory, Maxwell's Equations, Time Dilation, Lorentz transformation, Sagnac effect, Doppler effect, Cosmological redshift, Mass-Energy relationship, Particle acceleration, One-way light speed, Michelson-Morley experiment, Twin paradox

\section{INTRODUCTION}

The second postulate of Special Relativity (Einstein $1905[1,2,3])$, "the principle of the constancy of the velocity of light", stated: "in empty space, light is always propagated with a definite velocity $\mathrm{V}$ which is independent of the state of motion of the emitting body". This principle was experimentally validated by, the uniformity of the timing signature of the binary $\mathrm{x}$-ray pulsar system Her X1 [8], the measurement of the speed of gamma rays emitted by fast pions [11], and the laboratory experiments with moving optical elements [12]. However, the implicit inference in Special Relativity that the light velocity is also independent of the motion of the observer due to the "principle of relativity" [1], was never directly proven by any experiment. Other important theories of light velocity include the ether theory (Lorentz 1904 [6]) which is empirically equivalent to Special Relativity, and the emission theory (Ritz 1908 [9]) which contradicted the principle of constant light speed [10].

Starting from scratch, this paper mathematically derives a theoretic framework, "Asymmetry Theory", based only on the well-established "principle of the constancy of the velocity of light". Hence, all the experiments supporting this principle also support Asymmetry Theory. The key disagreement with Special Relativity is whether the light velocity is independent of the motion of the observer, which was never experimentally proved anyway.

First, a formula for the velocity of light is derived mathematically, which concludes that while the light always propagates at constant speed $c$ independent of the movement of the emitter, the velocity of light as detected by an observer is $\boldsymbol{c}-\boldsymbol{v}_{\boldsymbol{o}}(t), \boldsymbol{v}_{\boldsymbol{o}}(t)$ is the observer's velocity relative to the origin of the light (Note: not the emitter), i.e. the light velocity relative to the light origin is always constant $\boldsymbol{c}$, while the light velocity relative to the observer is $\boldsymbol{c}-\boldsymbol{v}_{\boldsymbol{o}}(t)$. The Sagnac effect $[15,16]$ is a clear proof of this formula, which is simply due to the different velocities of two light beams. This formula provides mathematical explanations of the problem of one-way light velocity, Abhorrence of starlight, and Michelson-Morleyexperiment [7]. For example, the one-way light velocity as measured by an observer is $\boldsymbol{c}-\boldsymbol{v}_{\boldsymbol{o}}(t)$, not $\boldsymbol{c}$. This 
asymmetry of light velocity should not be surprising. The propagation speed of a wave is independent of the motion of the emitter but its speed relative to an observer depends on the observer's motion. For an unrelated but helpful example, consider the sound wave from a supersonic jet vs an observer in the jet. The Doppler Effect [20] further shows that different observers detect the same light at varying frequencies depending on their velocities.

Secondly, the asymmetry between the times of light emission and observation is investigated to explain the phenomenon of "time dilation": when an observer moving away from a clock, the clock appears ticking slower and when the velocity approaches the light speed, he will see the clock coming to a stall. If he compares the observed clock with his clock, he will see a "time dilation" factor. This "time dilation" is due to the delay between the time of light emission as measured by the light source, $t_{s}$, and the observed time as measured by the observer, $t_{o}$, which results in a time scaling factor, $d t_{o} / d t_{s}$. A formula of this "observed" time dilation, distinguishing from the "real" time dilation in Special Relativity, is mathematically derived and the "twin paradox" is resolved.

The Doppler effect is simply a phenomenon of "observed" time dilation. A clock ticking slower/faster is equivalent to an "observed" lower/higher frequency. A general formula is mathematically derived from the "observed" time dilation, which covers traditional and transverse Doppler effects, Cosmological redshift, and time-varying velocities. This formula predicts no frequency shift for circular motion, supported by experiments $[13,14]$.

Maxwell's equations [5] provide the theoretic base and proof of Asymmetry Theory. A generalized form of Maxwell's wave equations for moving observers is mathematically derived from the original Maxwell equations, and shown covariant under the Galilean transformation. The formulas of the light velocity and Doppler effect in Asymmetry Theory can be mathematically derived from Maxwell's wave equations. With an interpretation of the velocity consistent with Barnett's experiment [18], the Lorentz force law is shown invariant under the Galilean transformation. Hence, the Lorentz transformation, which was formulated to keep the light velocity constant to any observer, is not required.

A set of formulas on the electrodynamics of participles is mathematically derived, which provides straightforward and mathematical explanations of why a particle is difficult to accelerate at high speed and why the mass of a body emitting photons changes.

This paper designed two experiments based on the predictions of Asymmetry Theory to further confirm the conclusion that the light velocity depends on the motion of observers. The first is to directly measure the travel times of a light beam to two detectors moving at the same speed in opposite directions. The second is to measure the momentum-to-acceleration ratios of a particle in an electromagnetic accelerator by reversing the direction of acceleration. Asymmetry Theory predicts an immediate jump of the ratio during the reversal of direction.

\section{THE VELOCITY OF LIGHT}

\section{A. Principle of Constant Light Velocity}

This paper is solely based on the mathematic derivation from the following principle [1].

Principle 1: In empty space, the light always propagates with a velocity $c$. independent of the state of motion of the emitting body.

Define $\mathrm{t}_{\mathrm{s}}$ as the time that the light was emitted; $\mathbf{S}\left(\mathrm{t}_{\mathrm{s}}\right)$ as the origin of the light emitted at $t_{s} ; t_{o}$ as the time an observer $\mathbf{O}$ observed the light from $\mathbf{S}\left(\mathrm{t}_{\mathrm{s}}\right) ; \mathbf{O}\left(\mathrm{t}_{\mathrm{o}}\right)$ as the position of $\mathbf{O}$ at $\mathrm{t}_{\mathrm{o}}$.

Note: The origin of the light $\mathbf{S}\left(\mathrm{t}_{\mathrm{s}}\right)$ is different from the position of the emitter. They always coincide at the emission time $t_{s}$ but may not after $t_{s}$. Imagine an emitter sends a photon at $t_{s}$ and immediately turns off. At $t_{s}$, the origin of light $\mathbf{S}\left(t_{s}\right)$ is the emitter's position. After $t_{s}$, the emitter may continue moving but its motion won't impact the previously emitted light according to Principle 1, which means $\mathbf{S}\left(\mathrm{t}_{\mathrm{s}}\right)$ may no longer coincide with the emitter. An unrelated but helpful example is the position of a supersonic airplane vs. the origin of the sound wave.

Principle 1 is represented with the general equation governing the velocity of light

$$
\boldsymbol{O}\left(t_{o}\right)-\boldsymbol{S}\left(t_{s}\right)=\boldsymbol{c}\left(t_{o}-t_{s}\right)
$$

First, let's consider in the reference frame $\mathbf{F}$ in which $\mathbf{S}\left(\mathrm{t}_{\mathrm{s}}\right)$ is static. Define $\boldsymbol{v}_{o}(\mathrm{t})$ as the velocity of the observer $\mathbf{O}$ relative to $\mathbf{S}\left(\mathrm{t}_{\mathrm{s}}\right)$. In $\mathbf{F}$, (1) becomes:

$$
\begin{aligned}
& \boldsymbol{O}\left(t_{s}\right)-\boldsymbol{S}\left(t_{s}\right)+\boldsymbol{O}\left(t_{o}\right)-\boldsymbol{O}\left(t_{s}\right)=c\left(t_{o}-t_{s}\right) \\
& \Rightarrow \boldsymbol{O}\left(t_{s}\right)-\boldsymbol{S}\left(t_{s}\right)+\int_{t s}^{t o} \boldsymbol{v}_{o}(t) d t=c\left(t_{o}-t_{s}\right) \\
& \Rightarrow \boldsymbol{O}\left(t_{s}\right)-\boldsymbol{S}\left(t_{s}\right)=\int_{t s}^{t o}\left(c-\boldsymbol{v}_{o}(t)\right) d t
\end{aligned}
$$

For the same light origin $\mathbf{S}\left(\mathrm{t}_{\mathrm{s}}\right)$ and observer $\mathbf{O}$, (2) is proved covariant under the Galilean transformations from F to different reference frames (Appendix. A). Hence, equation (2) holds for any reference frame.

\section{B. The light velocity/speed formula}

By definition, average speed $=$ distance / travel time. $\mathrm{We}$ have: 
Theorem 1: The average speed for a light emitted from $\mathbf{S}\left(\mathrm{t}_{\mathrm{s}}\right)$ to an observer $\mathbf{O}$ detecting it at $\mathrm{t}_{\mathrm{o}}$, is

$$
c_{\mathrm{so}}=\frac{\left|\int_{t s}^{t o}\left(\boldsymbol{c}-\boldsymbol{v}_{o}(t)\right) d t\right|}{t_{o}-t_{s}}
$$

Proof: It is obvious from (2) and the definition.

By definition, displacement $=$ integral of velocity. We have:

Lemma 1: If a light is emitted from $\mathbf{S}\left(\mathrm{t}_{\mathrm{s}}\right)$ to an observer $\mathbf{O}$ detecting it at $\mathrm{t}_{\mathrm{o}}$, the velocity of this light, $\boldsymbol{c}_{\mathrm{so}}(\mathrm{t})$, must satisfy, for any $t_{s}$ and $t_{o}$,

$$
\boldsymbol{O}\left(t_{s}\right)-\boldsymbol{S}\left(t_{s}\right)=\int_{t s}^{t o} \boldsymbol{c}_{s o}(t) d t
$$

Theorem 2: The velocity of a light emitted from $\mathbf{S}\left(\mathrm{t}_{\mathrm{s}}\right)$ as to an observer $\mathbf{O}$ with velocity $\boldsymbol{v}_{\mathrm{O}}(\mathrm{t})$, is

$$
c_{\text {so }}(\mathrm{t})=c-v_{\mathrm{o}}(\mathrm{t})
$$

Proof: From (2) and (4) we have:

$$
\int_{\mathrm{ts}}^{\text {to }} \boldsymbol{c}_{\text {so }}(\mathrm{t})=\int_{\mathrm{ts}}^{\mathrm{to}}\left(\boldsymbol{c}-\boldsymbol{v}_{\mathrm{o}}(\mathrm{t})\right) \mathrm{dt}
$$

For this equation to hold for any $t_{s}$ and $t_{o}$, we have:

$$
c_{\text {so }}(\mathrm{t})=\boldsymbol{c}-\boldsymbol{v}_{\mathrm{o}}(\mathrm{t})
$$

Note: (3) can also be directly derived from (5).

Corollary 1: The speed of light from $\mathbf{S}\left(\mathrm{t}_{\mathrm{s}}\right)$ to $\mathbf{O}\left(\mathrm{t}_{\mathrm{o}}\right)$ with velocity $v_{o}$ perpendicular to $\overrightarrow{\mathbf{S}\left(\mathrm{t}_{\mathrm{s}}\right) \mathbf{O}\left(\mathrm{t}_{\mathrm{s}}\right)}$ is

$$
c_{s o}=c^{*} \sqrt{1-v_{o}^{2} / c^{2}}
$$

This is a direct derivation from (5). It is important to note that the speed of light is $c$ times the Lorentz factor, which is formulated to force-fit the assumption of constant light velocity to observers according to Asymmetry Theory.

In summary,

1. The light speed/velocity is independent of the motion of the emitter.

2. When $v_{\mathrm{O}}(\mathrm{t})=0$ the light velocity is constant $\boldsymbol{c}$.

3. The velocity of light as to an observer is directly related to its velocity, specifically, $c-v_{\mathrm{o}}(\mathrm{t})$.

\section{One-way Light Speed \& M-M experiment}

Corollary 2: The round-trip speed of light from $\mathbf{S}\left(\mathrm{t}_{\mathrm{s}}\right)$ to $\mathbf{O}$ with velocity $v_{o}$ along the light path is

$$
c_{s o}=c^{*}\left(1-\frac{v_{o}^{2}}{c^{2}}\right)
$$

Proof: From (5), we have

$$
c_{s o}=\frac{2}{\frac{1}{c-v_{o}}+\frac{1}{c+v_{o}}}=c *\left(1-\frac{v_{o}^{2}}{c^{2}}\right)
$$

The round-trip speed of light $c_{s o} \approx c$ if $v_{o} \ll c$. If $v_{o}=460 \mathrm{~m} / \mathrm{s}$, the earth rotation speed, the difference is only $0.7 \mathrm{~mm} / \mathrm{s}$. Put it in the interferometer used in the Michelson-Morley-experiment [7], the difference is an undetectable 0.00005 fringe, which explains the null result.

As to the one-way speed of light, if $v_{o}=460 \mathrm{~m} / \mathrm{s}$, the measured difference from $c$ is $460 \mathrm{~m} / \mathrm{s}$ according to (5). Hence, formula (5) provides a straightforward explanation of the problem of measuring $c$ in one-way.

\section{The composition of velocity}

Assuming one light origin $\mathbf{S}\left(\mathrm{t}_{\mathrm{s}}\right)$, and two observers $\mathbf{O}$ and $\mathbf{O}^{\prime}$ with a relative velocity of $\boldsymbol{v}$. The velocities of $\mathbf{O}$ and $\mathbf{O}^{\prime}$ relative to $\mathbf{S}\left(\mathrm{t}_{\mathrm{s}}\right), \boldsymbol{v}_{o^{\prime}}(\mathrm{t})$ and $\boldsymbol{v}_{\mathrm{o}}(\mathrm{t})$, should follow

$$
v_{o^{\prime}}(\mathrm{t})-v_{\mathrm{o}}(\mathrm{t})=v
$$

From (5), the velocity of the light from $\mathbf{S}\left(\mathrm{t}_{\mathrm{s}}\right)$ to $\mathbf{O}$ and $\mathbf{O}^{\prime}, \boldsymbol{c}_{\text {so }}(\mathrm{t})$ and $\boldsymbol{c}_{\mathrm{so}}(\mathrm{t})$, should follow

$$
c_{\mathrm{so}^{\prime}}(\mathrm{t})=\boldsymbol{c}-\boldsymbol{v}_{\mathrm{o}^{\prime}}(\mathrm{t})=\boldsymbol{c}_{\mathrm{so}}(\mathrm{t})-\boldsymbol{v}
$$

The light velocity follows the addition of velocities.

\section{E. The Sagnac Effect}

The Sagnac effect [15] shows that two light beams, sent clockwise and counterclockwise around a closed path on a rotating disk, take different time intervals to travel the path, which contradicted the assumption that the light velocity is independent of the motion of the observer. Special Relativity attributed this contradiction to the rotating/accelerating frame $[4,17]$. The Sagnac effect can be extended to a FOG [16] with $\Delta \mathrm{t}=2 v L / \mathrm{c}^{2}$, where $v$ is the speed of the detector, $L$ is the length of the path.

Let $v_{o}^{+}, v_{o}^{-}$be the velocities of the detector to those two light beams, then $v_{o}^{+}-v_{o}^{-}=2 v$. According to (5), the light velocities as measured by the detector are $c-v_{o}^{+}$and $c-v_{o}^{-}$, respectively. Therefore,

$$
\Delta \mathrm{t}=\frac{L}{c-v_{o}^{-}}-\frac{L}{c-v_{o}^{+}}=\frac{2 v L}{\left(c-v_{o}^{-}\right)\left(c-v_{o}^{+}\right)} \approx \frac{2 v L}{c^{2}}
$$

In summary, the Sagnac Effect is the straightforward inference of the light velocity formula (5) in Asymmetry Theory. 


\section{F. Key angles and stellar aberration}

Aberration of starlight is a phenomenon of difference in the observed angle of starlight due to the velocity of the observer. FIG.1 shows key angles for the velocity of light, $\theta_{s}, \theta_{o}, \phi$, and $v_{\mathrm{s}}\left(\mathrm{t}_{\mathrm{s}}\right)$ denotes the velocity of the light source relative to the light origin $\mathbf{S}\left(\mathrm{t}_{\mathrm{s}}\right)$ at $\mathrm{t}_{\mathrm{s}}$. The stellar aberration is $\theta_{o}-\phi$. Assuming $v_{\mathrm{o}}(\mathrm{t})$ is constant $v_{\mathrm{o}}$, the relationship is

$$
\sin \left(\theta_{o}-\phi\right)=\frac{v_{o}}{c} \sin (\phi)
$$

When $\phi=90^{\circ}, \sin \left(\theta_{o}-\phi\right)=\frac{v_{o}}{c}$ and $\cos \left(\theta_{o}\right)=-\frac{v_{o}}{c}$, which are the same as Special Relativity [1], and not a coincidence considering (6).

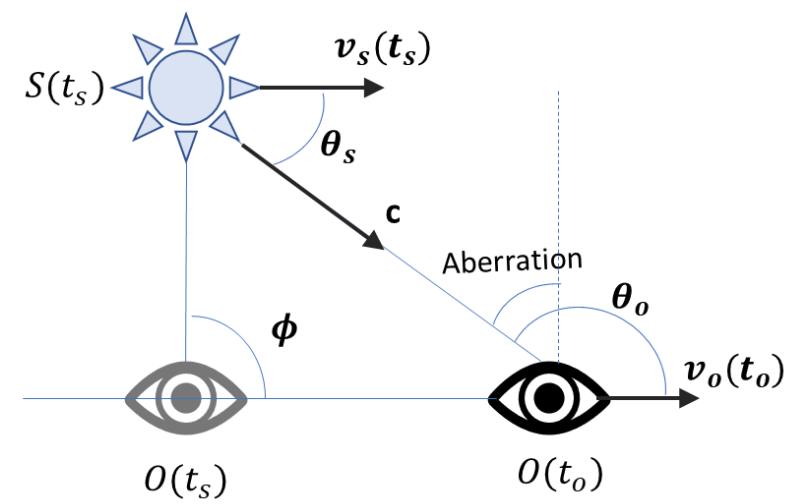

FIG. 1. Key angles and stellar aberration

\section{TIME DILATION}

\section{A. "Observed" time dilation}

The "observed time dilation" is defined as the observation timing difference between, $\mathrm{t}_{\mathrm{s}}$ and $\mathrm{t}_{\mathrm{o}}$, specifically, the scaling factor, $d t_{o} / d t_{s}$. From (1),

$$
\boldsymbol{O}\left(t_{o}\right)-\boldsymbol{S}\left(t_{s}\right)=\boldsymbol{c}\left(t_{o}-t_{s}\right)
$$

Perform an inner product of both sides, we have

$$
\left(\boldsymbol{O}\left(t_{o}\right)-\boldsymbol{S}\left(t_{s}\right)\right) \cdot\left(\boldsymbol{O}\left(t_{o}\right)-\boldsymbol{S}\left(t_{s}\right)\right)=c^{2}\left(t_{o}-t_{s}\right)^{2}
$$

Differentiate both sides as to $t_{s}$

$$
\begin{gathered}
\left(\boldsymbol{O}\left(t_{o}\right)-\boldsymbol{S}\left(t_{s}\right)\right) \cdot\left(\boldsymbol{v}_{o}\left(t_{o}\right) \frac{d t_{o}}{d t_{s}}-\boldsymbol{v}_{s}\left(t_{s}\right)\right)=c^{2}\left(t_{o}-t_{s}\right)\left(\frac{d t_{o}}{d t_{s}}-1\right) \\
\Rightarrow \frac{\left(\boldsymbol{O}\left(t_{o}\right)-\boldsymbol{S}\left(t_{s}\right)\right)}{c\left(t_{o}-t_{s}\right)} \cdot\left(\boldsymbol{v}_{o}\left(t_{o}\right) \frac{d t_{o}}{d t_{s}}-\boldsymbol{v}_{s}\left(t_{s}\right)\right)=c\left(\frac{d t_{o}}{d t_{s}}-1\right)
\end{gathered}
$$

Let $\mathrm{i}_{\text {os }}$ denote the unit vector in $\overrightarrow{\mathbf{S}\left(\mathrm{t}_{\mathrm{s}}\right) \mathbf{O}\left(\mathrm{t}_{\mathrm{s}}\right)}$, we have

$$
\boldsymbol{i}_{o s} \cdot \boldsymbol{v}_{o}\left(t_{o}\right) \frac{d t_{o}}{d t_{s}}-\boldsymbol{i}_{o s} \cdot \boldsymbol{v}_{s}\left(t_{s}\right)=c\left(\frac{d t_{o}}{d t_{s}}-1\right)
$$

Finally, the formula for observed time dilation is

$$
\frac{d t_{o}}{d t_{s}}=\frac{c-v_{s}\left(t_{s}\right) \cos \left(\theta_{s}\right)}{c-v_{o}\left(t_{o}\right) \cos \left(\theta_{o}\right)}
$$

When $v_{\mathrm{o}}(\mathrm{t}), v_{\mathrm{s}}(\mathrm{t})$ are constant $v_{o}, v_{s}$ in the same direction as $\boldsymbol{c}$, i.e. $\theta_{\mathrm{s}}, \theta_{\mathrm{o}}=0,(10)$ reduces to

$$
\frac{d t_{o}}{d t_{s}}=\frac{c-v_{s}}{c-v_{o}}
$$

This formula clearly explains "time dilation" as the observed difference of elapsed time. When $v_{\mathrm{o}}$ increases, the observed elapsed time $d t_{o}$ increases compared to the elapsed time $d t_{s}$ in the emitter, i.e. the clock appears ticking slower. Similarly, when $v_{\mathrm{o}}$ decreases, the clock appears ticking faster. When $v_{\mathrm{o}}$ approaches $c, d t_{o}$ approaches infinity, which means that the observer will see the clock coming to a stall.

\section{B. The "twin paradox"}

Now consider the scenario of "twin paradox". Assume a light source is at rest and an observer moves away from the same position and returns. Each records its time as $t_{s}$ and $t_{o}$, with the start times synchronized as $t_{s}=t_{o}=0$, and the return times as $t_{s}=t_{1}$ and $t_{o}=t_{1}{ }^{\prime}$, respectively.

The elapsed times recorded by each can be represented as $\int_{0}^{t_{1}} d t_{s}$ and $\int_{0}^{t_{1}{ }^{\prime}} d t_{o}$, respectively. Substituting $d t_{s}$ with $d t_{o}$ according to equation (10), we have

$$
\begin{aligned}
\int_{0}^{t_{1}} d t_{s} & =\int_{0}^{t_{1}{ }^{\prime}}\left(\frac{c-v_{o}\left(t_{o}\right) \cos \left(\theta_{o}\right)}{c}\right) d t_{o} \\
& =\int_{0}^{t_{1}{ }^{\prime}} d t_{o}-\frac{1}{c} \int_{0}^{t_{1}{ }^{\prime}} \cos \left(\theta_{o}\right) v_{o}\left(t_{o}\right) d t_{o} \\
& =\int_{0}^{t_{1}{ }^{\prime}} d t_{o}-\frac{1}{c} \oint_{L} \cos \left(\theta_{o}\right) d s,
\end{aligned}
$$

where $d$ s is the incremental distance of the observer over its path $\boldsymbol{L}$. Since $\boldsymbol{L}$ is a closed contour, $\oint_{L} \cos \left(\theta_{o}\right) d s=0$. We have

$$
\int_{0}^{t_{1}} d t_{s}=\int_{0}^{t_{1}{ }^{\prime}} d t_{o} \text { or } t_{1}=t_{1}{ }^{\prime}
$$

which means the elapsed times measured by $t_{s}$ and $t_{o}$ are always the same and independent of the movement. There is no "twin paradox" for the observed "time dilation" regardless of acceleration. 


\section{THE DOPPLER EFFECT}

\section{A. The general formula of the Doppler Effect}

The Doppler effect [20] is the change in frequency of the light to an observer who is moving relative to the light source. The Doppler Effect is the straightforward mathematical derivation from the observed time dilation. Because the total wavenumber emitted during the period $\mathrm{dt}_{\mathrm{s}}$ should be equal to that received during the period $\mathrm{dt}_{\mathrm{o}}$, we have the following equation:

$$
f_{o}\left(t_{o}\right) * d t_{o}=f_{s}\left(t_{s}\right) * d t_{s}
$$

Hence,

$$
\frac{f_{s}\left(t_{s}\right)}{f_{o}\left(t_{o}\right)}=\frac{d t_{o}}{d t_{s}}
$$

Substitute with the time dilation formula (10), we get the general formula of the Doppler effect:

$$
\frac{f_{s}\left(t_{s}\right)}{f_{o}\left(t_{o}\right)}=\frac{c-v_{s}\left(t_{s}\right) \cos \left(\theta_{s}\right)}{c-v_{o}\left(t_{o}\right) \cos \left(\theta_{o}\right)}
$$

When the velocities $v_{o}(t), v_{s}(t)$ are constants, $v_{o}, v_{s}$ and in the direction $\overline{\mathbf{S}\left(\mathrm{t}_{\mathrm{s}}\right) \mathbf{O}\left(\mathrm{t}_{\mathrm{o}}\right)}$, it reduces to the traditional formula of Doppler Effect [20]:

$$
\frac{f_{s}}{f_{o}}=\frac{c-v_{s}}{c-v_{o}}
$$

In summary, the general formula (12) applies to both the traditional and transverse Doppler effects, and also timevarying velocities.

Since the Doppler Effect is a direct result of the varying propagation delay of light, there is no frequency shift when the distance between the observer and the light source is constant, such as a circular movement. The relativistic Doppler Effect [19] predicts a frequency shift. Experiments $[13,14]$ agreed with Asymmetry Theory.

\section{B. The Cosmological red-shift}

Cosmological red-shift is traditionally believed to be the effect of the inflating Universe and different from the Doppler Effect [21]. Assuming $a(t)$ is the cosmic scale factor, the formula of cosmological red-shift is:

$$
\frac{f_{s}\left(t_{s}\right)}{f_{o}\left(t_{o}\right)}=\frac{a\left(t_{o}\right)}{a\left(t_{s}\right)}
$$

In Asymmetry Theory, cosmological red-shift is the same Doppler effect. Under the assumption of inflating Universe, the general equation (1) becomes:

$$
\boldsymbol{O}\left(t_{o}\right)-\boldsymbol{S}\left(t_{s}\right)=\int_{t s}^{t o} c / a(t) d t
$$

Following the similar derivation in III. A, we have the corresponding Doppler effect formula:

$$
\frac{f_{s}\left(t_{s}\right)}{f_{o}\left(t_{o}\right)}=\frac{c / a\left(t_{s}\right)-v_{s}\left(t_{s}\right) \cos \left(\theta_{s}\right)}{c / a\left(t_{o}\right)-v_{o}\left(t_{o}\right) \cos \left(\theta_{o}\right)}
$$

When $v_{s}\left(t_{s}\right), v_{o}\left(t_{o}\right) \ll c$

$$
\frac{f_{s}\left(t_{s}\right)}{f_{o}\left(t_{o}\right)} \approx \frac{a\left(t_{o}\right)}{a\left(t_{s}\right)}
$$

which is the same as (14). Hence, the cosmological redshift is a phenomenon of the Doppler Effect.

\section{MAXWELL'S EQUATIONS}

\section{A. Lorentz Force Law}

An electrical particle $\mathrm{P}$ with charge $\mathrm{q}$ in an electrical and magnetic field will experience the Lorentz force [22] of

$$
\mathbf{f}=\mathrm{q}^{*}(\mathrm{E}(\mathrm{x}, \mathrm{y}, \mathrm{z}, \mathrm{t})+\boldsymbol{v} \times \mathrm{B}(\mathrm{x}, \mathrm{y}, \mathrm{z}, \mathrm{t}))
$$

Traditionally, $v$ is defined as the velocity of $\mathrm{P}$ in any chosen reference frame. Barnett's Experiment of a rotating solenoid magnet [18] invalidated this definition. A cylindrical capacitor with a fine wire shorting its inner and outer conductors is coaxial with a solenoid magnet. In case 1 , the capacitor is rotating with angular velocity $\omega$, while the solenoid is at rest. Lorentz force is detected. In case 2, the capacitor is at rest, but the solenoid is rotating with $\omega$. No Lorentz force is detected. These results clearly showed that only the motion relative to the magnetic field generates Lorentz force. Barnett's experiment is explained if $v$ is measured as the velocity relative to the center of the magnetic field, the axis. Hence, in case $1, v=\omega^{*} \mathrm{r}$, while in case $2, \boldsymbol{v}=0$.

Consider the case there is a center, $\mathrm{S}$, which represents the magnetic field. $v$ can be defined as the velocity of $\mathrm{P}(\mathrm{x}, \mathrm{y}, \mathrm{z})$ relative to $\mathrm{S}\left(\mathrm{x}_{0}, \mathrm{y}_{0}, \mathrm{z}_{0}\right)$ :

$$
v=\frac{d(P-S)}{d t}
$$

Perform the Galilean transformation in a different reference frame moving with relative speed $\mathrm{v}$ in the $\mathrm{x}$ direction, $\mathrm{P}$ and $\mathrm{S}$ become $\mathrm{P}^{\prime}\left(\mathrm{x}^{\prime}, \mathrm{y}^{\prime}, \mathrm{z}^{\prime}\right)$ and $\mathrm{S}^{\prime}\left(\mathrm{x}_{0}^{\prime}, \mathrm{y}_{0}^{\prime}, \mathrm{z}_{0}^{\prime}\right)$. We have

$$
v^{\prime}=\frac{d\left(P^{\prime}-S^{\prime}\right)}{d t^{\prime}}=\frac{d(P-S)+d\left(\left(x^{\prime}-x\right)+\left(x_{0}^{\prime}-x_{0}\right)\right) i_{x}}{d t}=\boldsymbol{v}
$$

Let $\mathrm{E}^{\prime}, \mathrm{B}^{\prime}$ be the fields after transformation, we should have the same Lorentz force in both coordinates. Hence,

$$
\begin{gathered}
\mathrm{q}^{*}\left(\mathrm{E}^{\prime}\left(\mathrm{x}^{\prime}, \mathrm{y}^{\prime}, \mathrm{z}^{\prime}, \mathrm{t}^{\prime}\right)+v^{\prime} \times \mathrm{B}^{\prime}\left(\mathrm{x}^{\prime}, \mathrm{y}^{\prime}, \mathrm{z}^{\prime}, \mathrm{t}^{\prime}\right)\right)= \\
\mathrm{q}^{*}(\mathrm{E}(\mathrm{x}, \mathrm{y}, \mathrm{z}, \mathrm{t})+\boldsymbol{v} \times \mathrm{B}(\mathrm{x}, \mathrm{y}, \mathrm{z}, \mathrm{t}))
\end{gathered}
$$

Since $v^{\prime}=v$, for (18) to hold for any $v$, we have

$$
\mathrm{E}^{\prime}=\mathrm{E} \text { and } \mathrm{B}^{\prime}=\mathrm{B}
$$


In summary, The Lorentz force law is invariant under Galilean transformation and the electrical and magnetic fields don't change simply due to the transformation of coordinates.

\section{B. Generalized Maxwell Wave Equations}

Maxwell's standard wave equations [5] in a vacuum and charge-free space are:

$$
\begin{aligned}
& \nabla^{2} \mathrm{E}(\mathbf{r}, \mathrm{t})-\frac{1}{c^{2}} \frac{\partial^{2}}{\partial t^{2}} \mathrm{E}(\mathbf{r}, \mathrm{t})=0 \\
& \nabla^{2} \mathrm{~B}(\mathbf{r}, \mathrm{t})-\frac{1}{c^{2}} \frac{\partial^{2}}{\partial t^{2}} \mathrm{~B}(\mathbf{r}, \mathrm{t})=0
\end{aligned}
$$

In a reference frame static to the light origin, the clock everywhere, i.e. $t$, is in the same scale as the clock in the light origin, i.e. $t_{s}$ in Asymmetry Theory. Equation (20) can be rewritten as:

$$
\nabla^{2} \mathrm{E}\left(\mathbf{r}, \mathrm{t}_{\mathrm{s}}\right)-\frac{1}{c^{2}} \frac{\partial^{2}}{\partial t_{s}{ }^{2}} \mathrm{E}\left(\mathbf{r}, \mathrm{t}_{\mathrm{s}}\right)=0
$$

By solving this equation, the wave propagation velocity is constant c, consistent with Principle 1.

From the perspective of an observer, only the time it detects the light, i.e. $t_{o}$ in Asymmetry Theory, can be used to measure the wave propagation. Assume an observer with a velocity $v_{o}$ along the direction of the wave propagation. From equation (11), since $v_{s}=0$ for charge-free space, we have

$$
\frac{d t_{o}}{d t_{s}}=\frac{c}{c-v_{o}}
$$

Substitute $t_{s}$ with $t_{o}$ in (22), we have

$$
\nabla^{2} \mathrm{E}\left(\mathbf{r}, \mathrm{t}_{\mathrm{o}}\right)-\frac{1}{\left(c-v_{o}\right)^{2}} \frac{\partial^{2}}{\partial t_{o}{ }^{2}} \mathrm{E}\left(\mathbf{r}, \mathrm{t}_{\mathrm{o}}\right)=0
$$

Similarly,

$$
\nabla^{2} \mathrm{~B}\left(\mathbf{r}, \mathrm{t}_{\mathrm{o}}\right)-\frac{1}{\left(c-v_{o}\right)^{2}} \frac{\partial^{2}}{\partial t_{o}^{2}} \mathrm{~B}\left(\mathbf{r}, \mathrm{t}_{\mathrm{o}}\right)=0
$$

(24), (25) are the generalized Maxwell wave equations as to the observers moving in the direction of wave propagation. These equations are equivalent to the original Maxwell equations (20)(21), simply in another form using the time $t_{o}$ from the perspectives of the observer. When $v_{o}=0$, they reduce to the original Maxwell equations.

\section{Light velocity and Doppler Effect formulas}

The general solution to (24) is a linear superposition of waves of the form:

$$
\mathrm{E}\left(\mathbf{r}, t_{o}\right)=\mathrm{g}\left(2 \pi f_{o} t_{o}-\mathbf{k} \bullet \mathbf{r}\right)
$$

where $f_{o}$ is the frequency observed by the observer, $\mathrm{k}$ is the wave vector and $k=|\mathbf{k}|$ is the wavenumber. From (24), $f_{o}, k$ shall satisfy

$$
k^{2}-\frac{1}{\left(c-v_{o}\right)^{2}}\left(2 \pi f_{o}\right)^{2}=0
$$

Hence, the propagation velocity $c_{s o}$ is

$$
c_{s o}=\frac{2 \pi f_{o}}{k}=c-v_{o}
$$

which is the same formula of light velocity derived in (5).

The observed frequency $f_{o}$ is

$$
f_{o}=\left(c-v_{o}\right) * k / 2 \pi
$$

Since the emission frequency is $f_{s}=c * k / 2 \pi$, we have:

$$
\frac{f_{o}}{f_{s}}=\frac{c-v_{o}}{c}
$$

which is the same formula as the Doppler effect in (13).

In summary, the formulas of the light velocity and Doppler effect can be derived from the generalized Maxwell wave equations, which provide the theoretic base for Asymmetry Theory.

\section{The covariance of Maxwell's wave equations}

Perform the Galilean transformation of the generalized Maxwell wave equations from the reference frame $\mathbf{F}$ to a frame $\mathbf{F}^{\prime}$ moving with relative speed $v$ in the x-direction. For simplicity, assume the wave propagation is in the $\mathrm{x}$ direction. The generalized Maxwell wave equations for the observer $\mathrm{O}$ with the relative velocity $v_{o}$ in $\mathbf{F}$ is

$$
\nabla^{2} \mathrm{E}-\frac{1}{\left(c-v_{o}\right)^{2}} \frac{\partial^{2}}{\partial t_{o}^{2}} \mathrm{E}=0
$$

Let $v_{o}{ }^{\prime}$ be the relative velocity of observer $\mathrm{O}^{\prime}$ in $\mathrm{F}^{\prime}$, from equation (23) we have

$$
\frac{d t_{o}{ }^{\prime}}{d t^{\prime}}=\frac{c}{c-v_{o}{ }^{\prime}} \text { and } \frac{d t_{o}}{d t}=\frac{c}{c-v_{o}}
$$

Since $t^{\prime}=t$, we have

$$
\frac{d t_{o}}{c-v_{o}}=\frac{d t_{o}^{\prime}}{c-v_{o}^{\prime}}
$$

Since $\mathrm{E}^{\prime}=\mathrm{E}$ from (19) and $\nabla^{\prime}=\nabla$, substituting $\mathrm{t}_{\mathrm{o}}, \mathrm{v}_{\mathrm{o}}$ with $\mathrm{t}_{\mathrm{o}}{ }^{\prime}, \mathrm{v}_{\mathrm{o}}{ }^{\prime}$ in (28), w have

Similarly,

$$
\nabla^{\prime 2} \mathrm{E}^{\prime}-\frac{1}{\left(c-v_{o}^{\prime}\right)^{2}} \frac{\partial^{2}}{\partial t_{o}{ }^{2}} \mathrm{E}^{\prime}=0
$$

$$
\nabla^{\prime 2} \mathbf{B}^{\prime}-\frac{1}{\left(c-v_{o}\right)^{2}} \frac{\partial^{2}}{\partial t_{o}{ }^{2}} \mathbf{B}^{\prime}=0
$$

Therefore, the generalized Maxwell wave equations are covariant under the Galilean transformation. 


\section{ELECTRODYNAMICS}

Similar to Special Relativity $[1,2]$, a set of electrodynamics equations is mathematically derived from the light velocity formula (5) in Asymmetry Theory.

\section{A. Momentum to acceleration ratio}

It is well known that a particle becomes difficult to accelerate when its speed approaches $c$. This phenomenon is the direct result of the speed of the electromagnetic wave relative to the particle. According to the formula of light velocity (5), when the particle's velocity $v_{o}$ increases, the light velocity $c-v_{o}$ decreases, and correspondingly, the momentum gained by the particle decreases.

Assuming a particle has a mass $m$ and a velocity $v_{o}$, the momentum of the photons accelerating the particle is $\Delta P$, and the incremental velocity that the particle gains is $\Delta v_{o}$. Since the velocity of the photons relative to the particle is $c-v_{o}$, we have the following equation of momentum conservation for the case of perfectly inelastic collision

$$
\begin{aligned}
& \frac{\Delta P}{c}\left(c-v_{o}\right)=m^{*} \Delta v_{o} \\
\Rightarrow & \frac{\Delta P}{\Delta v_{o}}=m /\left(1-\frac{v_{o}}{c}\right)
\end{aligned}
$$

From (30), when $v_{o}$ increases, the momentum to acceleration ratio increases, and when $v_{o}$ approaches $c$, the ratio approaches infinity, i.e. the particle can no longer be accelerated.

The key prediction is that the momentum to acceleration ratio depends on not only the value but also the direction of the velocity $v_{o}$. Assume $v_{o}$ is $c / 2$ and the direction is reversed, a $3 \mathrm{x}$ ratio change is predicted. This prediction, different from Special Relativity [1], is utilized to design an experiment to validate Asymmetry Theory.

\section{B. The Mass-Energy relationship}

If all the photons are absorbed by the particle and let $\Delta \mathrm{m}$ denotes the incremental mass after the absorption, we have the following equation of momentum conservation:

$$
\begin{gathered}
\Delta P+m^{*} v_{o}=(m+\Delta m) *\left(v_{o}+\Delta v_{o}\right) \\
\Rightarrow \Delta P+m^{*} v_{o}=m^{*} v_{o}+\Delta m^{*} v_{o}+m^{*} \Delta v_{o}+\Delta m * \Delta v_{o}
\end{gathered}
$$

Omit the second-order $\Delta m^{*} \Delta v_{o}$ and substitute $m^{*} \Delta v_{o}$ with (29), we have

$$
\begin{aligned}
\Delta P= & \Delta m * v_{o}+\frac{\Delta P}{c} *\left(c-v_{o}\right) \\
& \Rightarrow \Delta P * c=\Delta m * c^{2}
\end{aligned}
$$

We derived the Mass-Energy relationship

$$
\Delta E=\Delta m * c^{2}
$$

This Mass-Energy relationship (32) is consistent with Special Relativity [2] and explains the mass change when a body emitting photons.

\section{Mass change of a moving particle}

Substitute $\Delta P$ in (31) with equation (29), we have

$$
\begin{gathered}
\\
m * \Delta v_{o} * \frac{v_{o}}{c-v_{o}}=\Delta m * v_{o} \\
\Rightarrow d m / m=d\left(\frac{v_{o}}{c}\right) /\left(1-\frac{v_{o}}{c}\right)
\end{gathered}
$$

Assuming a particle is accelerated from $v_{o}$ to $v_{o}{ }^{\prime}$ and all photons are absorbed during this acceleration, by integrating (33), we have:

$$
m\left(v_{o}{ }^{\prime}\right)=m\left(v_{o}\right) *\left(1-v_{o} / c\right) /\left(1-v_{o}{ }^{\prime} / c\right)
$$

\section{EXPERIMENTS DESIGN}

Two experiments are designed to conclusively confirm if the light velocity is independent of the motion of observers.

\section{A. Light velocity to moving observers}

A transient light source and two moving light sensors are in the same initial position and connected to the same precise digital scope with cables of equal length, see FIG. 2 . This setup is to avoid the synchronization argument by Special Relativity. A high-speed camera is an optional enhancement to capture the exact moments when the photon hits the sensors. Both sensors are moving at a constant speed $v$ but in opposite directions. Light is emitted when both sensors are in the same position. The detection times are measured. The light may be reflected multiple times with mirrors to increase the length of the light path $L$.

If the light velocity is independent of the motion of observers, both sensors shall detect the light at the same moment. Instead, Asymmetry Theory predicts a difference $\Delta t=2 v L /\left(c^{2}-v^{2}\right)$, same as the Sagnac effect. As long as there is a measurable time difference or a sequence of detections captured by the camera, the result will invalidate the assumption that the light velocity is independent of the motion of observers.

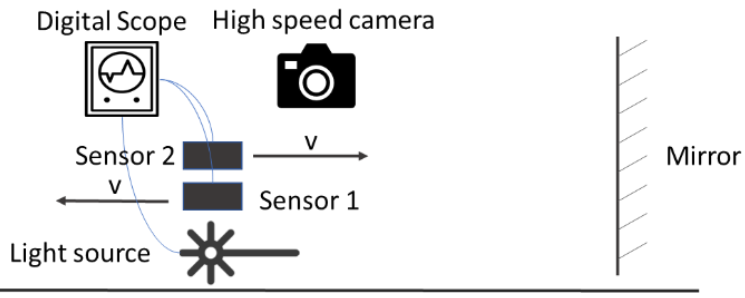

FIG. 2. Setup for the light velocity to moving observers 


\section{B. Asymmetry of momentum to acceleration}

The second experiment is based on the asymmetry of acceleration/deceleration of particles in a linear electromagnetic accelerator. Let's measure the ratio of the momentum applied by the accelerator to the acceleration of the particle, which is expected to decrease during the acceleration. First, the particle is accelerated to a high speed, say $0.5 c$. Then the direction of the accelerator is reversed to decelerate the particle. If Special Relativity is right, the direction reversal shouldn't have any immediate impact on the ratio. However, according to Asymmetry theory, the electromagnetic wave's speed relative to the particle suddenly changes from $0.5 c$ to $1.5 c$. Hence the momentum to acceleration ratio will experience a dramatic increase according to (29). The result should confirm which prediction is correct and if the light velocity is independent of the motion of observers.

\section{CONCLUSIONS}

Asymmetry Theory is derived mathematically based solely on the well-established principle of constant light velocity. It is comprehensive, self-consistent, and in harmony with all existing experiments. Compared to other theories, Asymmetry Theory provides straightforward and mathematical explanations of key physical phenomenons without any paradox.

The key conclusion is that the light velocity relative to an observer varies with the observer's motion. Two experiment designs based on the predictions of Asymmetry Theory are proposed, which should conclusively confirm the validity of this conclusion.

To avoid controversy, the physical meanings of Asymmetry Theory and its comparison with Special Relativity are discussed separately.

\section{ACKNOWLEDGEMENTS}

This research is sponsored by the International Education Foundation.

\section{APPENDIX}

\section{A. Galilean transformation of equation (2)}

The Galilean transformation relating the light origin $\mathbf{S}\left(\mathrm{t}_{\mathrm{s}}\right)$ static frame $\mathbf{F}$ to an inertial frame $\mathbf{F}^{\prime}$ moving with relative speed $\mathrm{v}$ in the $\mathrm{x}$-direction is,

$$
\begin{aligned}
\mathrm{x}^{\prime} & =\mathrm{x}-\mathrm{v} t \\
\mathrm{y}^{\prime} & =\mathrm{y} \\
\mathrm{z}^{\prime} & =\mathrm{z} \\
\mathrm{t}^{\prime} & =\mathrm{t}
\end{aligned}
$$

The light origin $\mathbf{S}\left(t_{s}\right)$ and observer $\mathbf{O}$ become $\mathbf{S}^{\prime}\left(t_{s}{ }^{\prime}\right)$ and $\mathbf{O}^{\prime}$ under the new coordinate $\mathbf{F}^{\prime}$. First, it is important to note that $\mathbf{S}\left(t_{s}\right)$ is static under $\mathbf{F}$ and hence moving with relative speed $-v$ under the new frame $\mathbf{F}^{\prime}$. We have

$$
\frac{d \boldsymbol{O}^{\prime}\left(t^{\prime}\right)}{d t^{\prime}}=\boldsymbol{v}_{o}(t)-v \text { and } \frac{d \boldsymbol{S}^{\prime}\left(t_{s}^{\prime}\right)}{d t^{\prime}}=-v
$$

Hence,

$$
\boldsymbol{v}_{\boldsymbol{o}}^{\prime}\left(t^{\prime}\right)=\frac{d \boldsymbol{O}^{\prime}\left(t^{\prime}\right)}{d t^{\prime}}-\frac{d \boldsymbol{S}^{\prime}\left(t_{s}^{\prime}\right)}{d t^{\prime}}=\boldsymbol{v}_{\boldsymbol{o}}(t)
$$

Secondly, the simple coordinate transformation shouldn't change the distance between the same light origin $\mathbf{S}\left(\mathrm{t}_{\mathrm{s}}\right)$ and observer $\mathbf{O}$, i.e.

$$
\boldsymbol{O}^{\prime}\left(t_{s}{ }^{\prime}\right)-\boldsymbol{S}^{\prime}\left(t_{s}{ }^{\prime}\right)=\boldsymbol{O}\left(t_{s}\right)-\boldsymbol{S}\left(t_{s}\right)
$$

Substitute (34), (35) into (2), we have

$$
\boldsymbol{O}^{\prime}\left(t_{s}{ }^{\prime}\right)-\boldsymbol{S}^{\prime}\left(t_{s}{ }^{\prime}\right)=\int_{t_{s}{ }^{\prime}}^{t_{o}{ }^{\prime}}\left(\boldsymbol{c}-\boldsymbol{v}_{\boldsymbol{o}}^{\prime}\left(t^{\prime}\right)\right) \mathrm{d} t
$$

Hence, (2) is covariant under Galilean transformation. 
[1] A. Einstein, On the Electrodynamics of Moving Bodies, Annalen der Physik, 17, 891-921 (1905).

[2] A. Einstein, Does the Inertia of a Body Depend Upon its Energy-Content?, Annalen der Physik, 323 (13): 639641 (1905).

[3] A. Einstein, On the Possibility of a New Test of the Relativity Principle, Annalen der Physik, 328 (6): 197 198 (1907).

[4] A. Einstein, Generalized theory of relativity, the anthology 'The Principle of Relativity', 94, University of Calcutta (1920).

[5] J. Maxwell, A Dynamical Theory of the Electromagnetic Field, Philos. Trans. R. Soc. Lond. $155,459-512$ (1865).

[6] H. Lorentz, Electromagnetic phenomena in a system moving with any velocity smaller than that of light, Proc. R. Neth. Acad. Arts Sci. 6: 809-831 (1904).

[7] A. Michelson and E. Morley, On the Relative Motion of the Earth and the Luminiferous Ether, Am. J. Sci. 34 (203): 333-345 (1887).

[8] K. Brecher, Phys. Rev. Lett., 39, 1051 (1977).

[9] W. Ritz, Recherches Critiques sur les Theories Electrodynamiques de Cl. Maxwell et de H.-A. Lorentz, Archives des Sciences physiques et naturelles 36: 209 (1908).

[10] J. G. Fox, Evidence Against Emission Theories, Am. J. Phys. 33 (1): 1-17 (1965).

[11] F. Alvaeger et al., Phys. Lett. 12, 260 (1964).

[12] G. C. Babcock et al., J. O. S. A. 54, 147 (1964).

[13] D.C. Champeney et al., Absence of Doppler shift for gamma ray source and detector on same circular orbit., Proc. Phys. Soc. 77, 350 (1961).

[14] H. W. THIM, Absence of the relativistic transverse Doppler shift at microwave frequencies, IEEE Trans. Instr. Measur. 52, 1660-1664 (2003).

[15] G. Sagnac, C. R. Acad. Sci. Paris 157, 708 (1913).

[16] R. Wang et al., Physics Letters A. 312 (1-2): 7-10 (2006).

[17] Langevin, Paul, Sur la théorie de la relativité et l'expérience de M. Sagnac, Comptes Rendus. 173: 831-834 (1921).

[18] S.J. Barnett, On Electromagnetic Induction and Relative Motion, Phys. Rev. 35, 323 (1912).

[19] D. Sher, The Relativistic Doppler Effect, J. R. Astron. Soc. Can. 62: 105-111 (1968).

[20] N. Giordano, College Physics: Reasoning and Relationships, Cengage Learning, 421-424 (2009).

[21] T. Koupelis et al., In Quest of the Universe, Jones \& Bartlett Publishers, 557 (2007).

[22] H. Lorentz, Versuch einer Theorie der electrischen und optischen Erscheinungen in bewegten Körpern, Leiden: E.J. Brill (1895). 